\title{
PHƯƠNG PHÁP GẦN ĐÚNG GIẢI BÀI TOÁN TRUYỀN SÓNG ƯNG DỤNG TRONG THĂM DÒ ĐỊA CHẤN
}

\author{
LÊ XUÂN QUẢNG
}

\begin{abstract}
In this work we consider two models of seismic waves. We use projection method and Ray - methods for solving the problems:

$$
\rho^{2} \frac{\partial^{2} U}{\partial t^{2}}=(\lambda+\mu) \operatorname{grad} \theta+\mu \Delta U+P \chi
$$
\end{abstract}

\section{1. Đặt vấn đề}

Các bài toán cơ học chất lỏng, chất khí, trường điện tử, lan truyền sóng địa chấn trong môi trường đàn hồi được đặc trưng bới phương trình động lực học sau:

$$
\rho^{2} \frac{\partial^{2} U}{\partial t^{2}}=(\lambda+\mu) \operatorname{grad} \theta+\mu \Delta U+P \chi .
$$

Phương trình (1) được gọi là phương trình Lame trong đó $\rho$ là mật độ môi trường, $\lambda, \mu$ là các hằng số đàn hồi Lame, $\Delta$ là toán tử Laplas, còn $U$ là vectơ chuyển dịch, xác định sự chuyển dịch của các phần tử môi trường trong không gian ba chiều theo biến thời gian $t$ dưới tác động của ngoại lực $\chi$.

Nhiều lớp bài toán khác nhau với các điều kiện biên khác nhau của phương trình (1) được ứng dụng rộng rãi trong thức tiễn đã được các nhà cơ học, toán học nghiên cứu cả định tính và phương pháp giải.

Trong bài báo này chúng ta chỉ xét 2 trường hợp riêng của phương trình (1) được ứng dụng cho việc nghiên cứu sự lan truyền sóng địa chấn.

\section{Bài toán 1}

Xét phương trình truyền sóng

$$
\frac{\partial^{2} P}{\partial t^{2}}-q(t) P=\frac{\partial^{2} P}{\partial s^{2}} \quad(0 \leq t \leq s),
$$

với các điều kiện 


$$
\begin{gathered}
P(t, t)=\frac{1}{2} \int_{t}^{\infty} q(\tau) d \tau, \\
\lim _{s \rightarrow \infty} P(t, s)=0, \\
P(t, s)=0 \text { với } t>s .
\end{gathered}
$$

Định lý 1. Phương trình (2.1) với các điều kiện (2.2), (2.3), (2.4) có thể chuyên về dạng phương trình tích phân sau:

$$
\begin{gathered}
P(t, s)=\frac{1}{2} \int_{\frac{1}{2}(t+s)}^{\infty} q(\tau) d \tau+\frac{1}{2} \int_{t}^{\frac{1}{2}(t+s)} q(\tau) d \tau \int_{t+s-\tau}^{s+\tau-t} P(\tau, u) d u \\
-\frac{1}{2} \int_{\frac{1}{2}(t+s)}^{\infty} q(\tau) d \tau \int_{\tau}^{s+\tau-t} P(\tau, u) d u
\end{gathered}
$$

Chứng minh. Đầu tiên ta xét phương trình

$$
\frac{\partial^{2} Z}{\partial t^{2}}-\frac{\partial^{2} Z}{\partial s^{2}}=F(t, s)
$$

Giả sứ $V(t, s, \tau)$ là nghiệm của phương trình thuần nhất:

$$
\frac{\partial^{2} V}{\partial t^{2}}-\frac{\partial^{2} V}{\partial s^{2}}=0
$$

thỏa mãn điều kiện:

$$
\left.V\right|_{t=0}=0,\left.\frac{\partial V}{\partial t}\right|_{t=0}=F(\tau, s)
$$

khi đó dễ dàng thấy rằng hàm $Z(t, s)=\int_{t}^{\infty} V(\tau-t, s, \tau) d \tau$ thóa mãn (2.6). Vì $V(t, s, \tau)=\frac{1}{2} \int_{s-t}^{s+t} F(\tau, u) d u$ nên

$$
Z(t, s, \tau)=\frac{1}{2} \int_{s+t-\tau}^{s+\tau-t} F(\tau, u) d u
$$


Bây giờ chúng ta viết phương trình (2.1) dưới dạng:

$$
\frac{\partial^{2} P}{\partial t^{2}}-\frac{\partial^{2} P}{\partial s^{2}}=q(t) P
$$

và coi như vế phải là hàm đã biết. Sứ dụng công thức $(2.7)$ và các điều kiện $(2.2)$, (2.4) ta có:

$$
P(t, s)=\frac{1}{2} \int_{\frac{1}{2}(t+s)}^{\infty} q(\tau) d \tau-\frac{1}{2} \int_{t}^{\infty} d \tau \int_{s+t-\tau}^{s+\tau-t} d(\tau) p(\tau, u) d u
$$

Bây giờ một lần nữa sử dụng điều kiện (2.4). Nếu $s+t-\tau>\tau$ có nghĩa $\tau<\frac{1}{2}(t+s)$ thì $u>\tau$ và $P(\tau, u) \neq 0$ còn nếu $s+t-\tau<\tau$ có nghĩa $\tau<\frac{1}{2}(t+s)$ thì $u<\tau$ và $P(\tau, u)=0$. Từ đó phương trình (2.8) có dạng (2.6). Định lý được chứng minh.

Định lý 2. Nếu $q(t)$ khá vi và thóa mãn diều kiện:

$$
\int_{t}^{\infty} \tau|a(\tau)| d \tau=\delta_{1}(t)<\infty
$$

thi phuơng trình (2.6) có nghiệm duy nhất đàng thời đó là nghiệm của bài toán (2.1) - (2.4).

Trước hết ta xấp xỉ nghiệp của phương trình (2.6) bằng quá trình lặp sau:

$$
\begin{aligned}
P_{0}(t, s) & =\frac{1}{2} \int_{\frac{1}{2}(t+s)}^{\infty} q(\tau) d \tau \\
P_{m}(t, s) & =\frac{1}{2} \int_{t}^{\frac{1}{2}(t+s)} q(\tau) d \tau \int_{t+s-\tau}^{s+\tau-t} P_{m-1}(\tau, u) d u+ \\
& +\frac{1}{2} \int_{\frac{1}{2}(t+s)}^{\infty+\tau-t} q(\tau) d \tau \int_{\tau}^{\infty} P_{m-1}(\tau, u) d u \quad(m=1,2, \ldots)
\end{aligned}
$$

Chúng ta có các đánh giá sau: 


$$
\begin{aligned}
\left|P_{0}(t, s)\right| & \leq \frac{1}{2} \int_{\frac{1}{2}(t+s)}^{\infty}|q(\tau)| d \tau=\frac{1}{2} \delta\left(\frac{s+t}{2}\right) \\
\left|P_{1}(t, s)\right| & \leq \frac{1}{2} \int_{t}^{\frac{1}{2}(t+s)}|q(\tau)| \int_{t+s-\tau}^{s+\tau-t} \frac{1}{2} \delta\left(\frac{\tau+u}{2}\right) d u \\
& +\frac{1}{2} \int_{\frac{1}{2}(t+s)}|q(\tau)| d \tau \int_{\tau}^{\infty} \frac{1}{2} \delta\left(\frac{\tau+u}{2}\right) d u \\
& \leq \frac{1}{2} \delta\left(\frac{t+s}{2}\right) \int_{t}^{\infty} \tau|q(\tau)| d \tau=\frac{1}{2} \delta\left(\frac{t+s}{2}\right) \delta(t) .
\end{aligned}
$$

Theo công thức truy hồi chúng ta sẽ nhận được:

$$
\left|P_{m}(t, s)\right| \leq \frac{1}{2} \delta\left(\frac{\tau+u}{2}\right) \frac{\delta_{1}^{m}}{m !} \quad(m=0,1,2, \ldots)
$$

Từ đó suy ra

$$
P(t, s)=\sum_{m=0}^{\infty} P_{m}(t, s)
$$

là hội tụ tuyệt đối trong khoảng $0 \leq a \leq t<s$, và

$$
P(t, s) \leq \frac{1}{2} e^{\delta_{1}(t)} \delta\left(\frac{t+s}{2}\right) \text { thóa mãn phương trình (2.6). }
$$

Bây giờ chúng ta chứng minh $P(t, s)$ thỏa mãn bài toán $(2.1)-(2.4)$. Đễ chứng minh điều đó ta chỉ cần chứng minh:

(i) $\quad \int_{t}^{\infty}|P(t, s)| d s<\infty$.

(ii) $\int_{t}^{\infty}\left|\frac{\partial P(t, s)}{\partial s}\right| d s<\infty$.

(iii) $\int_{t}^{\infty}\left|\frac{\partial^{2} P}{\partial s^{2}}(t, s)\right| d s<\infty$.

(iv) $\lim _{s \rightarrow \infty} P(t, s)=\lim _{s \rightarrow \infty} \frac{\partial P}{\partial s}(t, s)=0$.

Thực vậy, trước hết ta có đánh giá: 


$$
\begin{aligned}
\int_{t}^{\infty} \delta(\tau) d \tau & =\int_{t}^{\infty} d \tau \int_{t}^{\infty}|q(x)| d x=\int_{t}^{\infty}|q(x)| d x \int_{t}^{x} d \tau \\
& =\int_{t}^{\infty}(x-t)|q(x)| d x \leq \delta_{1}(t)
\end{aligned}
$$

do vậy

$$
\int_{t}^{\infty}|P(t, s)| \leq \frac{1}{2} e^{\delta_{1}(t)} \int_{t}^{\infty} \delta\left(\frac{t+s}{2}\right) d s=e^{\delta_{1}(t)} \int_{t}^{\infty} \delta(u) d u=e^{\delta_{1}(t)} \delta_{1}(t) .
$$

Bây giờ ta lấy đạo hàm hai vế của (2.6)

$$
\begin{aligned}
\frac{\partial P}{\partial t}= & -\frac{1}{4} q\left(\frac{t+s}{2}\right)-\frac{1}{2} \int_{t}^{\frac{t+s}{2}} q(\tau) P\left(\tau, s_{t}-\tau\right) d \tau \\
& -\frac{1}{2} \int_{t}^{\infty} q(\tau) P(\tau, s+\tau-t) d \tau \quad(0<t<s) .
\end{aligned}
$$

Đối với $\frac{\partial p}{\partial s}$ cũng có đánh giá tương tự (2.14).

Từ đánh giá (2.12) và (2.14) ta có:

$$
\begin{aligned}
|P(t, s)| \leq & \frac{1}{4}\left|q\left(\frac{t+s}{2}\right)\right|+\frac{1}{4} e^{\delta_{1}(t)} \delta\left(\frac{t+s}{2}\right) \int_{t}^{\frac{t+s}{2}} q(\tau) d \tau \\
& +\frac{1}{4} e^{\delta_{1}(t)} \int_{t}^{\infty}|q(\tau)| \delta\left(\frac{2 \tau_{s}-t}{2}\right) d \tau \\
& \leq \frac{1}{4} q\left(\left|\frac{t+s}{2}\right|\right)+\frac{1}{2} e^{\delta_{1}(t)} \delta(t) \delta\left(\frac{t+s}{2}\right) .
\end{aligned}
$$

Từ các đánh giá trên có thể chứng minh (i) $\rightarrow$ (iii) và từ phương trình (2.6) chứng minh $\lim _{(t+s) \rightarrow \infty} P(t, s)=0$. 
Như vậy quá trình lặp $P_{m}(t, s)$ đã cho chúng ta thuật toán giải gần đúng bài toán (2.1) - (2.4). Chuổi (2.11) hội tụ tuyệt đối với tốc độ cao cho phép chúng ta tìm $P(t, s)$ dễ dàng.

\section{Bài toán 2} nhất.

Giải gần đúng phương trình truyền sóng địa chấn trong môi trường đồng

Bây giờ chúng ta xét môi trường truyền sóng sau:

$$
(\lambda+\mu) U_{j, i j}+\mu U_{j, j}+\lambda, i U_{j, j}+\mu, j\left(U_{i, j}+U_{j, i}\right)=P U_{i, t t}
$$

và tìm nghiệm phương trình (3.1) dưới dạng

$$
u_{i}\left(x_{m}, t\right)=U_{i}\left(x_{m}\right) F\left(t-\tau\left(x_{m}\right)\right),
$$

trong đó $U_{i}\left(x_{m}\right)$ f là hệ số tần số và $\tau\left(x_{m}\right)$ hàm chuyển dịch pha sẽ được xác định sau còn $F$ là hàm giải tích Hilbert thỏa mãn điều kiện:

$$
\begin{gathered}
d F^{(n)}(\theta) / d \theta=F^{(n-1)}(\theta), \\
F^{n}(\theta)=g^{n}(\theta)+i h^{n}(\theta),
\end{gathered}
$$

với $g^{n}(\theta)$ và $h^{n}(\theta)$ thỏa mãn:

$$
h(\theta)=\int_{-\infty}^{\infty} \frac{g(\delta)}{\delta-\theta} d \delta .
$$

Ví dụ: nếu lấy $F^{0}(\theta)=H(\theta)$ (hàm Heaviside)

$$
H(\theta)=\left\{\begin{array}{ll}
0 & \theta \leq 0 \\
1 & \theta>0
\end{array} \quad \text { thì } \quad F^{(n)}=\left\{\begin{array}{ll}
0 & \theta \leq 0 \\
\frac{\theta^{n}}{n !} & \theta>0
\end{array} .\right.\right.
$$

Chú ý rằng với $F$ thỏa mãn $(3.3),(3.4)$ thì $F^{(n)} \gg F^{(n-1)}$

Từ (3.2) lấy đạo hàm chúng ta thu được:

trong đó

$$
F^{\prime \prime} N_{i}\left(U_{m}\right)-F^{\prime} M_{i}\left(U_{m}\right)+F L_{i}\left(U_{m}\right)=0,
$$

$$
\begin{gathered}
M_{i}\left(U_{m}\right)=(\lambda+\mu)\left[U_{j, i} \tau_{i j}+U_{j, j} \tau_{i}+U_{i} \tau_{i j}\right]+\mu\left[2 U_{i, j} \tau_{i j}+U \tau_{i j}\right] \\
+\lambda_{i} U_{j} \tau_{i j}+\mu_{i j}\left(U_{i} \tau_{i j}+U_{j} \tau_{i}\right)
\end{gathered}
$$




$$
L_{i}\left(U_{m}\right)=(\lambda+\mu) U_{j, i}+\mu U_{i, j j^{\prime}}+\mu_{i j}\left(U_{i, j}+U_{j, i}\right) .
$$

Theo (3.5) $F^{\prime \prime} \gg F^{\prime} \gg F$ thì có thể coi

$$
N_{i}\left(U_{m}\right)=0, \quad M_{i}\left(U_{m}\right)=0 .
$$

Từ $N_{i}\left(U_{m}\right)=0$ cho ta

$$
\left(\frac{\lambda+\mu}{r h o} \tau, i \tau, j+\frac{\mu}{\rho} \tau, k \tau, k \partial_{i, j}-\partial_{i, j}\right) U_{j}=0
$$

hay

$$
\left(\bar{\Gamma}_{i j}-\partial_{i j}\right) U_{j}=0
$$

với $\Gamma_{i j}=\frac{\lambda+\mu}{\rho} \tau, i \tau, j+\frac{\mu}{\rho} \tau, k \tau, k \delta_{i j}$.

Từ thực tế $\tau, j=N_{j} / C$, trong đó $N_{j}$ là pháp tuyến của pha sóng đổi hướng của $U_{j}$ còn pha của vận tốc $C$ có thể xác định, nghiệm bài toán tìm $U_{j}$ có thể dẫn về việc tìm vectơ riêng của ma trận $\bar{\Gamma}_{i j}$ sau

$$
\left(\bar{\Gamma}_{i j}-G \delta_{i j}\right) g_{i}=0,
$$

trong đó $G$ là ma trận riêng thỏa mãn $G=1$, còn $g_{i}$ là vectơ riêng tương ứng ma trận $G$. Tốc độ pha có thể tìm từ điều kiện $G=1$ còn các tần số $U_{j}$ tỷ lệ với vectơ riêng $g_{i}$. Các giá trị riêng có thể tìm từ điều kiện:

$$
\operatorname{det}\left(\bar{\Gamma}_{i j}-G \delta_{i j}\right) g_{i}=0 .
$$

Với môi trường đồng nhất chúng ta có thễ tìm vectơ dưới dạng:

$$
D g_{i}=\bar{\Gamma}_{i j} g_{i}=\frac{\lambda+\mu}{\rho} \tau, i\left(\tau_{i j} g_{i}\right)+\frac{\mu}{\rho} \tau, k \tau, k g_{i},
$$

với $\left(\tau_{j} g_{i}\right) \neq 0$. Các vectơ riêng khác tìm trong điều kiện vuông góc với $\tau, i$ và song song $g_{i}$

$$
g_{i} \| \tau, i \text { và } \perp \tau, i
$$

Đầu tiên ta chọn $g_{i}=N_{i}$ và phương trình:

$$
G=\alpha^{2} \tau, k \tau, k=1,
$$

với $\alpha\left(x_{m}\right)=\sqrt{\frac{\lambda+2 \mu}{\rho}}$. Sau đó xác định

$$
U_{i}\left(x_{m}\right)=A\left(x_{m}\right) N_{i}\left(x_{m}\right) .
$$


Các giá trị $A\left(x_{m}\right)$ được xác định sau.

Tiếp tục cho $g_{i} \perp \tau, i$ ta lại có phương trình:

$$
G=\beta_{\tau, k}^{2} \tau, k=1,
$$

với $\beta\left(x_{m}\right)=\sqrt{\frac{\mu}{\rho}}$,

và $U_{i}\left(x_{m}\right)=B\left(x_{m}\right) g_{i}^{(1)}\left(x_{m}\right)+C\left(x_{m}\right) g_{i}^{(1)}\left(x_{m}\right)$, với $B\left(x_{m}\right)$ và $C\left(x_{m}\right)$ sẽ được xác định sau.

Bây giờ chúng ta đi xác định các hệ số $A\left(x_{m}\right), B\left(x_{m}\right)$ và $C\left(x_{m}\right)$ từ điều kiện $M_{i}\left(U_{m}\right)=0$. Qua một vài phép biến đổi và sử dụng điều kiện:

$$
\begin{aligned}
& \tau_{, i} g_{j}^{(1)}=0 \\
& g_{i, j}^{(1)} g_{j}^{(1)}=0 \\
& \tau_{, i j} g_{i}^{(1)}=-\tau_{j, i} g_{i, j}^{(1)} .
\end{aligned}
$$

Ta có phương trình xác định $A\left(x_{m}\right), B\left(x_{m}\right)$ và $\left(C\left(x_{m}\right)\right.$ như sau:

$$
\begin{aligned}
& \left(A A^{*} \rho \alpha^{2} \tau, i\right)_{, i}=0 \\
& 2 \rho \beta^{2} B{ }_{, i} \tau_{, i}+\rho \beta^{2} \tau_{, j j}+\left(\rho \beta^{2}\right)_{, i} \tau_{, i} B=0 \\
& 2 \rho \beta^{2} C_{, i} \tau_{j}+\rho \beta^{2} \tau_{, j j}+\left(\rho \beta^{2}\right)_{, i} \tau_{, i} C=0
\end{aligned}
$$

Như vậy chúng ta đã có sơ đồ để tính toán nghiệm của bài toán 2 . Vấn đề đánh giá độ xấp xỉ của nghiệm còn là vấn đề cần phải nghiên cứu thêm.

\section{TÀI LIÊU THAM KHẢO}

1. I. I. Gurvich, Phân tầng địa chấn. Moskva, 1976 (tiểng Nga).

2. B. M. Levitan, Lý thuyét hàm chuyển dịch suy rộng. Nhà xuất bản Khoa học, Moskva, 1973 (tiếng $\mathrm{Nga}$ ).

3. N. Bleistein, Mathematical method for ware phenomera. Academic Press, New York, 1984.

4. V. Cerveny, Seimic ray theory, 1994.

5. Lê Xuân Quảng, Phuơng pháp chiếu lặp giải phưong trình tích phân kỳ dị Fredholm loại III. Tạp chí Tin học và Điều khiển học, T. 10, số4 (1994).

Viện Công nghệ thông tin

Trung tâm KHTN và CNQG

Nhận bài ngày 20-1-1996 